\title{
Possibility to Interfere with Coronavirus RNA Replication Analyzed by Resonant Recognition Model
}

\author{
Irena Cosic ${ }^{1}$, Drasko Cosic ${ }^{1}$, Ivan Loncarevic ${ }^{2}$
}

\author{
${ }^{1}$ AMALNA Consulting, Black Rock, 3193, Australia \\ ${ }^{2}$ QuantBioRes - QBR A/S, Copenhagen, 2860, Denmark \\ Running Title: Analyzes of Coronavirus Viral RNA Replication
}

\begin{abstract}
To be able to design vaccine or even a cure for COVID-19, it is particularly important to understand how SARS-CoV-2, as a single stranded RNA virus, is multiplied within host cells and which factors are controlling this multiplication. Here, we have analyzed the process of coronavirus RNA replication within host cell with the aim to find out the characteristics of this process. For that purpose, we have utilized the Resonant Recognition Model (RRM), which is biophysical model capable of identifying parameters (frequencies) related to specific macromolecular (protein, DNA, RNA) functions and/or interactions. The RRM model is unique with its capability to directly analyze interactions between amino acid macromolecules (proteins) and nucleotide macromolecules (DNA, RNA). Using the RRM model, we have identified parameters that characterize two steps in coronavirus RNA replication i.e., initiation of replication and replication by itself. These parameters can be used in our future research to design peptides, that will be able to interfere with either or both of those processes.
\end{abstract}

Keywords: COVID-19, SARS-CoV-2, Coronavirus, Coronavirus RNA Replication, Resonant Recognition Model

\section{Introduction}

Current COVID-19 pandemic is caused by SARSCoV-2 virus, which belongs to group of singlestranded RNA viruses, more specifically coronaviruses. Coronaviruses are widely spread in nature, mostly infecting animals, but some can infect humans as well, usually with mild or non-existent symptoms [1]. However, there have been so far three instances where coronaviruses had infected humans causing severe symptoms, including SARS (Severe Acute Respiratory Syndrome, 2003), MERS (Middle East Respiratory Syndrome, 2012) and COVID-19 (2019-nCoV or SARS-CoV-2, 2019). While SARS and MERS outbreaks were within relatively limited areas of population in China and Middle East respectively, SARS-CoV-2 caused worldwide pandemic. In addition, SARS and MERS have been less infectious, but with higher mortality rate, while coronavirus SARS-CoV-2 is more infectious, but with lower mortality rate [2].

With the worldwide COVID-19 pandemic there is question if it is possible to design coronavirus vaccine or even cure. Firstly, it is particularly important to understand how SARS-CoV-2, as single-stranded RNA virus, is multiplied within host cells and which factors are controlling this multiplication. Infection begins when the viral spike protein attaches to its complementary host cell receptor. After attachment, a protease of the host cell cleaves and activates the receptor-attached spike protein. Depending on the host cell protease available, this cleavage and activation process allows the virus to enter the host cell [3].
On entry into the host cell, the virus particle is uncoated, and its genome enters the cell cytoplasm [4]. The coronavirus RNA genome has a 5' methylated cap and a $3^{\prime}$ polyadenylated tail, which allows viral RNA to attach to the host cell's ribosome for translation [4]. The host ribosome translates the initial overlapping open reading frame of the virus genome and forms a long polyprotein. The polyprotein has its own proteases, which cleave the polyprotein into multiple non-structural proteins [4]. Thus, there are two points to attack the virus, either to prevent its entry into the host cell, or to prevent its replication within the host cell. Here, we will concentrate to prevent replication within the host cell. Once, when the virus has entered the host cell, the most appropriate chance to prevent and find cure for the further infection is to prevent viral RNA translation. It is well known for initiation of RNA replication 5' and 3' noncoding parts of RNA could be critical in controlling RNA replication, as pointed out in ref [5]: "the nucleotide sequence of the 5' noncoding region of messenger RNAs is particularly interesting because it may contain the signals for processing and modification of mRNA, such as its cleavage from precursor and 'capping' (the addition of the $5^{\prime}$ terminal $\mathrm{m} 7 \mathrm{Gppp}$ ) and possible recognition and binding sites for ribosomes and initiation factors". Thus, we propose that interfering with RNA replication regulation through 5' noncoding segment could be possible way of preventing RNA virus replication.

Activation of 5' and 3' noncoding control segments of RNA is done by binding with specific RNA related 
proteins. One possible approach to understand this interaction process is to investigate function of argonaute proteins which are key players in smallRNA-guided gene regulation. Argonaute proteins are highly specialized small-RNA-binding proteins that coordinate downstream gene-silencing events and thus are considered to be the key components of RNA silencing pathways [6]. Recent work has made progress in understanding of argonaute-mediated gene-silencing principles, such as the effects on mRNA translation and decay, but has also implicated the argonaute proteins in several other cellular processes, such as transcriptional regulation and splicing [7]. In summary, argonaute proteins which consist of groups: AGO1, AGO2, AGO3 and AGO4, are highly specialized small-RNA-binding proteins driven by small RNAs to complementary target mRNAs, where they act together with protein binding partners to interfere with translation of target mRNAs. During virus infection individual functions of the mammalian antiviral RNA interference (RNAi) and micro-RNA (miRNA) effector proteins like argonautes (AGO1-AGO4) have been investigated showing that $\mathrm{AGO} 4$ has uniquely antiviral function in mammalian cells and has evolved to obtain antiviral RNAi [8].

In addition, there are number of RNA-dependent RNA polymerases that use RNA as their template for synthesis of a new strand of RNA. For instance, number of RNA viruses (such as poliovirus) use this type of enzyme to replicate their genetic material [9]. Also, RNA-dependent RNA polymerase is part of the RNA interference pathway in many organisms [10]. Of particular interest is viral replicase as well, which is enzyme that coordinates and mediates process of RNA synthesis [11].

On the other hand, there is number of 294 antibodies against SARS-CoV-2 mostly neutralizing SARSCoV-2 by blocking its binding to ACE2 receptor, where the most prevalent of those antibodies is IGHF3-53 [12]. Besides the fact that IGHF3-53 antibody can prevent viral spike protein interaction with ACE2 receptor, we will investigate here its possible interaction with RNA regulatory 5' segment and its possible role in preventing viral replication.

Baring all this in mind, we propose that interfering with RNA replication regulation through RNA directed transcriptase and viral replicase or through 5 , signal sequences within non-coding region of mRNA could be possible way of preventing RNA virus replication. Here, we have utilized our own biophysical Resonant Recognition Model (RRM) [13-20], which is capable of directly analyzing interaction between proteins and RNA [21-22], to analyze interaction between argonaute proteins, antibody IGHF3-53 and related RNA regulatory 5, signal sequences within non-coding region of mRNA with the aim to find out characteristic frequencies for initiation of viral replication. In addition, we have analyzed RNA replication through RNA directed transcriptase and viral replicase to identify characteristics of RNA viral replication by itself. Once when these RRM characteristics are identified, it is possible to further utilize the RRM model to design peptides, which would be able to interfere with initiation of viral replication and/or directly interfere with viral replication to possibly prevent virus multiplication within the host cell.

\section{Methods \\ Resonant Recognition Model}

The Resonant Recognition Model (RRM) is biophysical, theoretical model that can analyze interactions between proteins and their targets, which could be other proteins, DNA, RNA, or small molecules. The RRM has been previously published in detail within number of publications [13-20]. The RRM model is based on the findings that certain periodicities (frequencies) within the distribution of energy of delocalized electrons along protein backbone are critical for protein biological function and/or interaction with their targets. The distribution of delocalized electrons energies is calculated by assigning each amino acid specific physical parameter representing the energy of delocalized electrons of each amino acid. Consequently, the spectral characteristics of such energy distribution (signal) are calculated using Fourier Transform. This means that the linear numerical signal representing the distribution of energies along the protein is transformed into the frequency domain and is characterized by number of different frequencies containing all information from the original signal. Comparing such spectra using cross-spectral function for proteins, which are sharing the same biological function/interaction, it has been shown that they share the same frequency within the spectrum of free energy distribution along the macromolecule [13-20]. Peak frequencies in such multiple cross-spectral function present common frequency components for all macromolecular sequences compared. The comprehensive analysis done so far confirms that all macromolecular sequences, with the common biological function and/or interaction, have common frequency component, which is specific feature for the observed biological function/interaction [13-20]. Thus, each specific macromolecular biological function/interaction within macromolecule is characterized by specific RRM frequency.

Each biological function is driven by proteins that selectively interact with other proteins, DNA/RNA regulatory segments or small molecules. Through extensive use of RRM model, it has been shown that proteins and their targets share the same matching 
RRM characteristic frequency [13-24]. The matching of periodicities within the distribution of energies of free electrons along the interacting proteins can be regarded as the resonant recognition and as such is highly selective. Thus, the RRM frequencies characterize not only protein function, but also recognition and interaction between protein and its targets: proteins (receptors, binding proteins, and inhibitors), DNA/RNA regulatory segments or small molecules. In addition, it has been also shown that interacting macromolecules have opposite phases at their characteristic RRM recognition frequency [1320]. Every frequency can be presented by one sinusoid characterized with its three parameters: frequency, amplitude, and phase. The phase is presented in radians and can be between $-\pi$ and $+\pi$ (3.14 and +3.14$)$. The phase difference of or about $\pi$ (3.14) is considered to be opposite phase. The phase value can be presented in the phase circle where it is visually easier to observe phase differences.

The RRM model is unique approach, where it is possible to analyze interactions directly computationally between amino acid sequences (proteins) and nucleotide sequences (DNA and RNA), based only on matching frequencies within free electron energy distribution along these macromolecules. However, for the comparison of characteristic frequencies between proteins and DNA/RNA macromolecules, it is required to adjust for the difference in distances between amino acids

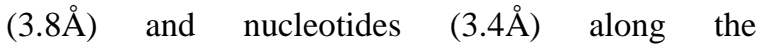
macromolecular backbone. These adjustments are made on nucleotide sequences spectrum, so the result could be compared with frequency calculations made for the proteins. These calculations enable the RRM to be the unique model capable of analyzing and directly comparing activities and interactions of proteins, DNA and RNA by identifying their characteristic frequencies, as tested, and described in number of previous publications [13-16,21-22].

Once the characteristic frequency for biological function and/or interaction of the macromolecule is identified, it is possible to design new peptides/proteins with the desired RRM frequency components and consequently with desired biological functions and/or interactions [13-16,25-30]. This design approach has already been successfully applied and experimentally tested in the design of FGF analogue [25], HIV envelope protein analogue
[26-28] and peptide to mimic myxoma virus oncolytic function [29-30].

As viruses are mutating their proteins, DNA and/or RNA very quickly, it is extremely hard to design general cure or vaccine. This explains why current approaches based on macromolecular homology are not successful enough for different viral strains. However, even when viruses are mutating so often and so quickly, they will still keep their specific functionality. It is important to understand that the RRM model is particularly efficient when it is applied to viruses, because the RRM model identifies the common characteristic parameter(s) for all mutated viruses, related to specific common virus protein's biological function. Thus, the RRM analysis of viruses does not depend on virus mutations, as long as they keep their functionality. This RRM approach has been experimentally tested on the example of HIV virus, which is continuously mutating, but all isolates keep the same functionality, as well as common RRM characteristic frequency [26-28].

\section{Results}

Here, we have focused on analysis of coronavirus RNA virus replication after virus enters the host cell. At that moment protease of the host cell cleaves and activates spike protein and the complete viral RNA becomes uncoated with its RNA genome entering the cell cytoplasm [4]. This is the critical point for further viral multiplication within the host cell. If it is possible to interfere at this critical point to disable viral multiplication it would prevent the development of related disease. The viral multiplication can be prevented by either interfering with initiation of viral RNA replication or directly by interfering viral RNA replication by itself.

Signal sequence within 5' non-coding region of mRNA is particularly interesting for initiation of viral RNA replication [5]. When we have analyzed 5' signal sequences within non-coding region of viral mRNA from different SARS coronavirus isolates, from UniProt database (MN938384.1, MN975262.1, MN985325, MN988668.1, MN988713, MN994467, MN994468, MN997409, MT072688.1, NC_045512.2), using the RRM, the most prominent common RRM frequency has been found at $\mathrm{f} 1=0.0234 \pm 0.0043$, as presented in Figure 1 . 


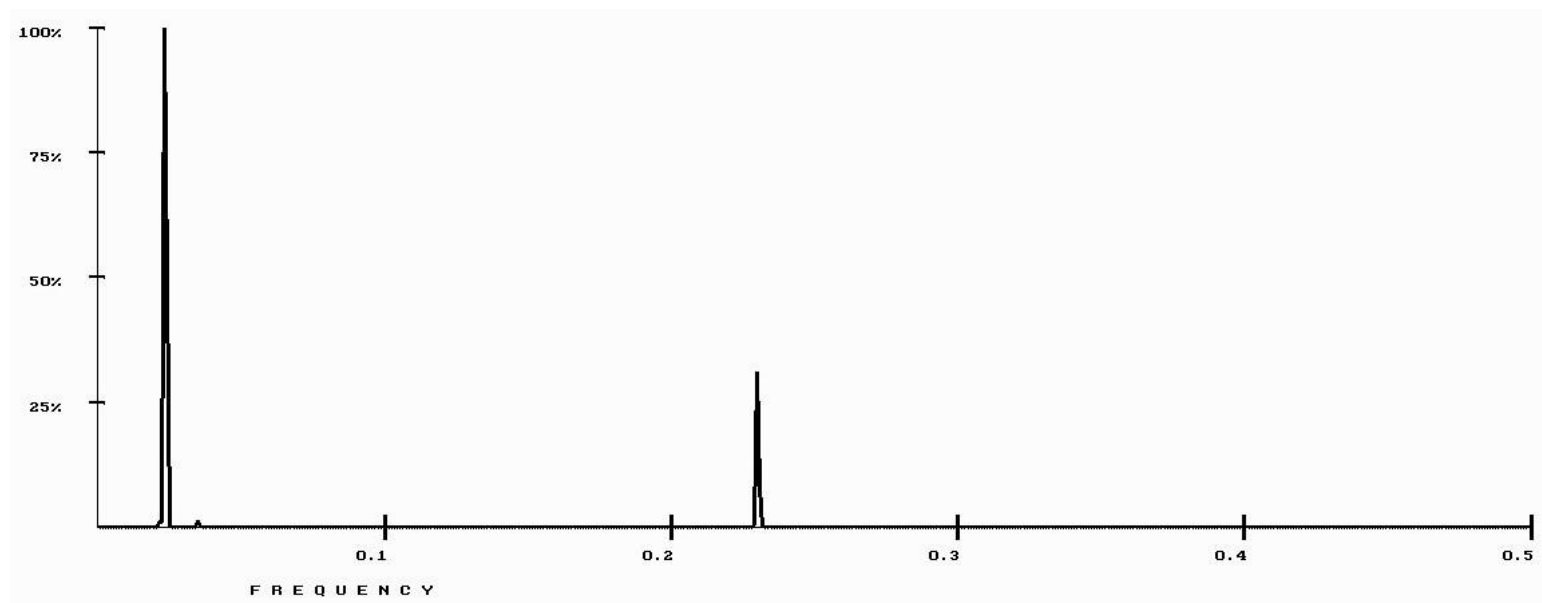

Figure 1. RRM cross-spectrum of 5' signal sequences within non-coding region of viral mRNA different SARS coronavirus isolates with common characteristic frequency at $\mathrm{f} 1=0.0234 \pm 0.0043$.

This common frequency f1 could be proposed to characterize functionality of 5 ' signal sequence, which allows viral RNA to attach to the host cell ribosome and is critical for initiation and control of viral RNA replication [5].

In addition, as it has been pointed out in Introduction, argonaute proteins (AGO1, AGO2, AGO3, AGO4) have a roll in mRNA translation, transcriptional regulation, and splicing [6-7]. In particular, AGO4 protein has uniquely antiviral function in mammalian cells [8]. Thus, we propose that argonaute (AGO4) protein function is related to activation of 5 ' signal sequences within non-coding region of viral mRNA. When we compared 5' signal sequences within noncoding region of viral mRNA from different SARS coronavirus isolates with mammalian $\mathrm{AGO} 4$ proteins, from UniProt database (Q9ZVD5, Q5ZMW0, Q9HCK5, Q8CJF8, Q9SDG8, Q0JF58, Q4KLV6), using the RRM, the same even more prominent common RRM frequency has been found at $\mathrm{f} 1=0.0234 \pm 0.0043$, as presented in Figure 2 .

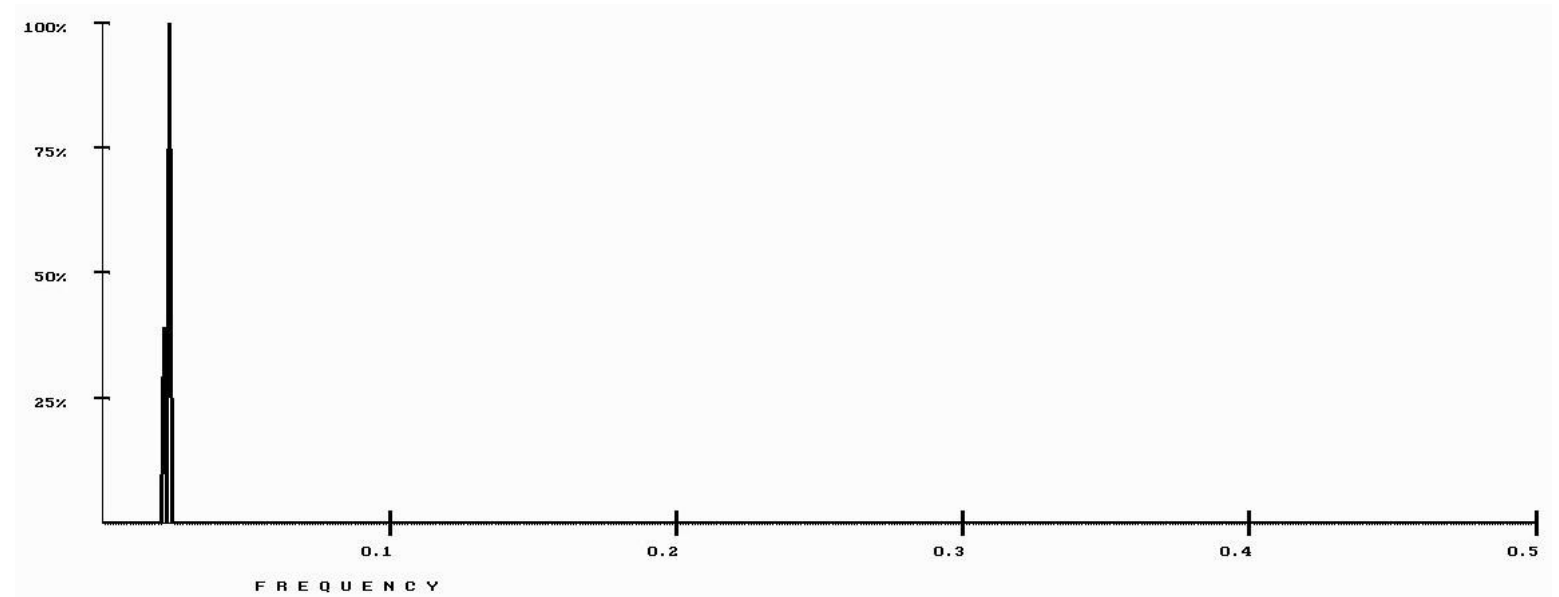

Figure 2. RRM cross-spectrum of 5' signal sequences within non-coding region of viral mRNA from different SARS coronavirus isolates and mammalian AGO4 proteins with common characteristic frequency at $\mathrm{f} 1=0.0234 \pm 0.0043$.

It is interesting to note that there is one unique common characteristic frequency for 5 , signal sequences within non-coding region of viral mRNA and argonaute (AGO4) proteins indicating the possibility that initiation of viral RNA replication involves host AGO4 proteins and viral 5' signal sequences within non-coding region of viral mRNA.

On the other hand, as explained in Introduction, there is large number of antibodies against SARS-CoV-2, where the most prevalent one is IGHF3-53 [12]. Besides the fact that IGHF3-53 antibody can prevent viral spike protein interaction with ACE2 receptor, we have investigated here possible interaction of IGHF3-53 antibody with viral RNA regulatory 5' signal sequences within non-coding region of viral mRNA as possible pathway for preventing initiation of viral replication.

When we have compared IGHF3-53 antibody with 
previously analyzed 5' signal sequences within noncoding region of viral mRNA different SARS coronavirus isolates and mammalian $\mathrm{AGO} 4$ proteins, from UniProt database (P01767), using the RRM, the same RRM frequency of $f 1=0.0234 \pm 0.0043$, became even more prominent, as presented in Figure 3.

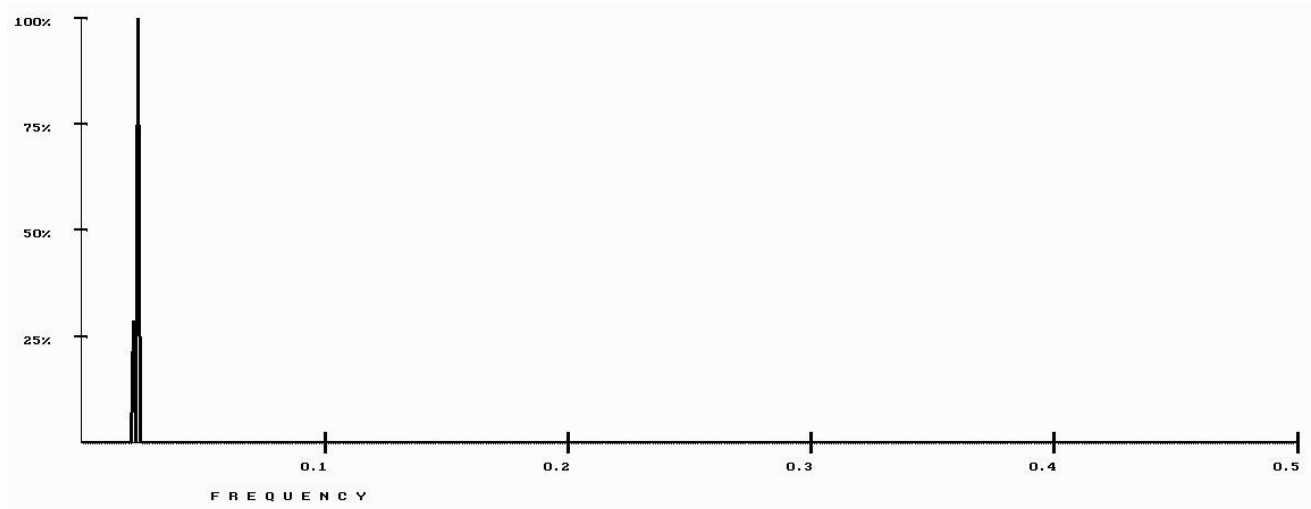

Figure 3. RRM cross-spectrum of IGHF3-53 antibody, 5' signal sequences within non-coding region of viral mRNA different SARS coronavirus isolates and mammalian AGO4 proteins with common characteristic frequency at $\mathrm{f} 1=0.0234 \pm 0.0043$.

This result indicates that IGHF3-53 antibody may be also able to interfere with activation of viral 5' signal sequences within non-coding region of viral mRNA and mimic activity of host AGO4 proteins, and thus prevent initialization of viral RNA replication. This result can be significant as we can use RRM frequency $\mathrm{f} 1=0.0234 \pm 0.0043$ as the basis to design peptides that can prevent initialization of viral RNA replication.
Another way to interfere with viral RNA replication is to prevent RNA dependent RNA polymerase and/or RNA replicase activity, which is critical for replication of viral RNA [9-11]. When we have analyzed RNA dependent RNA polymerase and RNA replicase, from UniProt database (Q02382, P25328, Q07048 and P35928, P20126, P36304, P20127, P10358, P20128, P28477, P59595), using the RRM, the most prominent RRM frequency was identified at $\mathrm{f} 2=0.0713 \pm 0.0011$, as presented in Figure 4 .

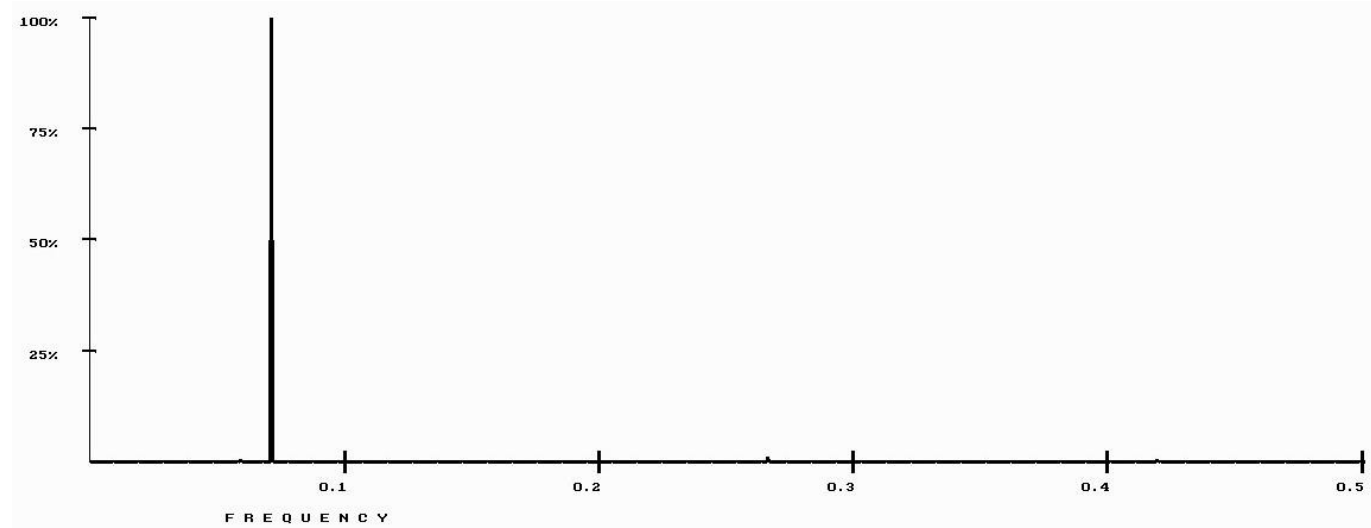

Figure 4. RRM cross-spectrum of RNA dependent RNA polymerase and RNA replicase proteins with common characteristic frequency at f2 $=0.0713 \pm 0.0011$

This frequency $\mathrm{f} 2=0.0713 \pm 0.0011$ is characterizing RNA dependent RNA polymerase and RNA replicase activity, i.e., replication of viral RNA.

Baring all above in mind, we propose that by interfering with frequency $\mathrm{f1}$, we can prevent initiation of viral RNA replication or by interfering with frequency $\mathrm{f} 2$, we can prevent viral RNA replication by itself. Using either or both of those ways, we propose that it would be possible to develop cure for coronaviruses. One possibility is to design peptides, using RRM model, that will be able to interfere with either or both of those activities

To achieve this, we have firstly identified two RRM characteristic frequencies: $\mathrm{f} 1=0.0234+0.0043$ characterizing initiation of viral RNA replication and f2 $=0.0713 \pm 0.0011$ characterizing viral RNA replication by itself. As described in RRM methodology, it is possible to design peptides having only the characteristic frequency and phase related to targeted protein. Thus, we have identified phases for all participants involved in initiation of RNA replication, as presented in Figure 5, within phase 
circle: for 5' signal sequences within non-coding region of viral mRNA from different SARS coronavirus isolates with phase of $-1.74 \mathrm{rad}$ in green, for IGHF3-53 antibody with phase of $+2.53 \mathrm{rad}$ in blue and for human AGO4 protein with phase of $+2.43 \mathrm{rad}$ in red. It can be easily observed from Figure 5, that phases for human AGO4 and IGFH3-53 antibody are very close to each other and almost opposite to phase for 5' signal sequences within noncoding region of viral mRNA from different SARS coronavirus isolates indicating that both $\mathrm{AGO} 4$ and IGHF3-53 interact with 5' signal sequences within non-coding region of viral mRNA during the process of initiation of RNA replication.

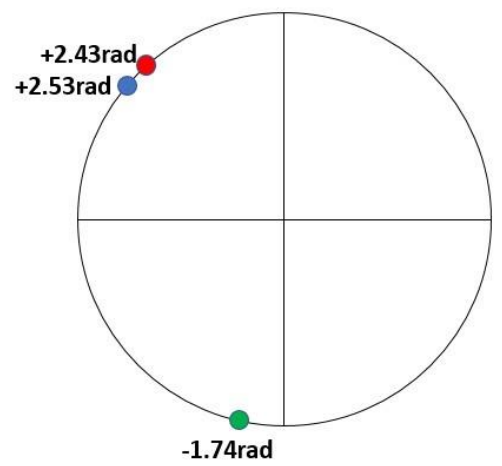

Figure 5. Phase circle at frequency of $\mathrm{fl}=0.0234$ for 5 ' signal sequences within non-coding region of viral mRNA from different SARS coronavirus isolates with phase of $-1.74 \mathrm{rad}$ in green, for IGHF3-53 antibody with phase of +2.53rad in blue and for human AGO4 protein with phase of $+2.43 \mathrm{rad}$ in red. It can be easily observed that phases for human AGO4 and IGFH3-53 antibody are very close to each other and almost opposite to phase for 5' signal sequences within non-coding region of viral mRNA from different SARS coronavirus isolates indicating that both AGO4 and IGHF353 interact with 5 ' signal sequence during the process of initiation of RNA replication.

In similar fashion, the phase for characteristic RRM frequency $\mathrm{f} 2$ has been identified to be at $+2.11 \mathrm{rad}$. Having identified the characteristic frequency f1 for initiation of RNA replication and frequency $\mathrm{f} 2$ characterizing viral RNA replication, as well as relevant phases at these frequencies, it will be possible to design peptides that can interfere with one and/or the other process in RNA replication and thus could be considered as possible cure for number of coronavirus related diseases.

\section{Discussion and Conclusion}

Here, we have analyzed the process of coronavirus RNA replication within host cell with the aim to find out the characteristics of this process. These characteristics can be used further in design of peptides possibly capable to interfere with this process and prevent viral RNA replication within host cell. For that purpose, we have utilized the Resonant Recognition Model (RRM), which is biophysical model capable of identifying parameters (frequencies) related to specific macromolecular (protein, DNA, RNA) function and/or interaction. The RRM model is unique with its capability to directly analyze interactions between amino acid macromolecules (proteins) and nucleotide macromolecules (DNA, RNA).

Using the RRM model, we have identified parameters that characterize two steps in RNA replication i.e., initiation of replication and replication by itself. Within the first step, we have identified RRM frequency $\mathrm{f} 1=0.0234 \pm 0.0043$, which is common for all 5' signal sequences within non-coding region of viral mRNA different SARS coronavirus isolates. As these 5' signal sequences are critical for initiation of viral RNA replication, we propose that this frequency $\mathrm{f} 1$ is characterizing initiation of viral RNA replication. In addition, we have found that the same frequency f1 is common with host's argonaute AGO4 proteins, which are key components of RNA silencing pathways [6] and thus we propose the possibility that negative control of initiation of viral RNA replication involves host's AGO4 proteins. We have also found that IGHF3-53 antibody shares the same common frequency $\mathrm{fl}$ with 5' signal sequences within non-coding region of viral mRNA different SARS coronavirus isolates and mammalian AGO4 proteins and thus we propose that IGHF3-53 antibody also have a role in negative control of initiation of viral RNA replication. As both AGO4 and IGHF3-53 antibody are proteins involved in negative control of initiation of viral RNA replication, sharing the same RRM characteristic frequency fl with 5' non-coding signal sequence, but with opposite phase, is confirming that frequency $\mathrm{f} 1$ is critical for negative control of initiation of viral RNA replication within host cell.

Within the second step of our analysis, we have identified RRM frequency $\mathrm{f} 2=0.0713 \pm 0.0011$, which is common to RNA dependent RNA polymerase and RNA replicase, and thus is proposed to characterize the process of viral RNA replication by itself.

Having identified the characteristic frequency $\mathrm{f} 1=0.0234 \pm 0.0043$ for initiation of RNA replication and frequency $\mathrm{f} 2=0.0713 \pm 0.0011$ characterizing viral RNA replication, we propose that by interfering with frequency $\mathrm{fl}$, we can prevent initiation of viral RNA replication and by interfering with frequency $\mathrm{f} 2$, we can prevent viral RNA replication. One possible approach would be to design peptides, using the RRM model, that will be able to interfere with either or both of these processes. This design will be a matter of our future research. 


\section{Contributions}

Conceptualization, I.C.; Methodology, I.C. and D.C.; Software, D.C.; Resources I.L.; WritingOriginal Draft Preparation-Review and Editing, I.C., D.C. and I.L.

\section{Competing Interests}

Authors declare they have no competing interests.

\section{Human/Animal Involvement}

Authors declare that there were no human participants nor any animal involvement in this study.

\section{Funding}

This research received no external funding.

\section{Acknowledgement}

The authors would like to thank Miss Amy Cosic for proofreading this manuscript.

\section{References}

1. Wu F, Zhao S, Yu B, Chen YM, Wang W, Song ZG, Hu Y, Tao ZW, Tian JH, Pei YY, Yuan ML, Zhang YL, Dai FH, Liu Y, Wang QM, Zheng JJ, Xu L, Holmes EC, Zhang YZ: A new coronavirus associated with human respiratory disease in China. Nature, 2020 Feb 3; doi: 10.1038/s41586-0202008-3

2. Makin S: How Coronaviruses Cause Infection from Colds to Deadly Pneumonia. Scientific American 2020 Feb 5.

3. Simmons G, Zmora P, Gierer S, Heurich A, Pöhlmann S: Proteolytic activation of the SARS-coronavirus spike protein: cutting enzymes at the cutting edge of antiviral research. Antiviral Research, 2013; 100(3), 605-614, doi 10.1016/j.antiviral.2013.09.028

4. Fehr AR, Perlman S: Coronaviruses: an overview of their replication and pathogenesis. Methods in Molecular Biology Springer, 2015; 1282, 1-23, doi: 10.1007/978-1-4939-2438 7_1.

5. Baralle E, Brownlee GG: AUG is the Only Recognisable Signal Sequence in the $5^{\prime}$ Non-coding Regions of Eukaryotic mRNA. Nature, 1978; 274, 84-87.

6. Cenik ES, Zamore PD: Argonaute proteins. Current Biology, 2011; 21(12), 446-449, doi: 10.1016/j.cub.2011.05.020.

7. Ender C, Meister G: Argonaute Proteins at a Glance. Journal of Cell Science, 2010; 123, 1819-1823, doi 10.1242/jcs.055210.

8. Adiliaghdam F, Basavappa M, Saunders TL, Harjanto D Prior JT, Cronkite DA, Papavasiliou N, Jeffrey KL: A Requirement for Argonaute 4 in Mammalian Antiviral Defense. Cell Reports Volume, 2020; 30(6), 1661-2054, doi 10.1016/j.celrep.2020.01.021.

9. Hansen JL, Long AM, Schultz SC: Structure of the RNAdependent RNA polymerase of poliovirus. Structure, 1997; 5(8), 1109-1122, doi: 10.1016/S0969-2126(97)00261-X.

10. Ahlquist P: RNA-dependent RNA Polymerases, Viruses, and RNA Silencing. Science, 2002; 296(5571), 1270-1273, doi: 10.1126/science.1069132.

11. Ziebuhr J: The Coronavirus Replicase. Corona Replication and Reverse Genetics, Nature Publishing Group, 2005; 287, 57-94.

12. Yuan M, Liu H, Wu NC, Lee CCD, Zhu X, Zhao F, Huang D, Yu W, Hua Y, Tien H, Rogers TF, Landais E, Sok D, Jardine JG, Burton DR, Wilson IA: Structural basis of a public antibody response to SARS-CoV-2. Science, 2020; 1-11, doi: 10.1126/science.abd.2321(2020).

13. Cosic I: Macromolecular Bioactivity: Is it Resonant Interaction between Macromolecules? -Theory and
Applications. IEEE Trans on Biomedical Engineering, 1994; 41, 1101-1114

14. Cosic I: Virtual spectroscopy for fun and profit Biotechnology, 1995; 13, 236-238.

15. Cosic I: The Resonant Recognition Model of Macromolecular Bioactivity: Theory and Applications. Basel Birkhauser Verlag, 1997

16. Cosic I: Resonant Recognition Model of Protein Protein and Protein DNA Recognition, in Bioinstrumentation and Biosensors. ed by Wise D. Marcel Dekker Inc., New York, 1990; 475-510.

17. Cosic I, Cosic D: Macromolecular Resonances. In Bandyopadhyay A., Ray K. (eds) Rhythmic Oscillations in Proteins to Human Cognition. Studies in Rhythm Engineering. Springer, Singapore, 2021; 1, 11-45, doi: 10.1007/978-981-15-7253-1 1.

18. Cosic I, Cosic D, Lazar K: Analysis of Tumor Necrosis Factor Function Using the Resonant Recognition Model. Cell Biochemistry and Biophysics, 2015; doi: 10.1007/s12013015-0716-3.

19. Cosic I, Paspaliaris V, Cosic D: Analysis of Protein-Receptor on an Example of Leptin-Leptin Receptor Interaction Using the Resonant Recognition Model. MDPI Appl. Sci., 2019; 9, 5169, doi:10.3390/app9235169.

20. Cosic I, Cosic D, Loncarevic I: RRM Prediction of Erythrocyte Band3 Protein as Alternative Receptor for SARS-CoV-2. MDPI Appl. Sci., 2020; 10, 4053, doi: 10.3390/app10114053.

21. Cosic I, Lazar K, Cosic D: Cellular Ageing - Telomere Telomerase and Progerin analysed using Resonant Recognition Model. MD-Medical Data, 2014; 6(3), 205-209.

22. Cosic I, Cosic D, Lazar K: Is it possible to predict electromagnetic resonances in proteins, DNA and RNA? Nonlinear Biomedical Physics, 2015; 3, doi: 10.1140/s40366015-0020-6.

23. Cosic I, Cosic D, Loncarevic I: New Concept of Small Molecules Interaction with Proteins - An Application to Potential COVID-19 Drugs. International Journal of Sciences, 2020; 9(9), 16-25, doi: 10.18483/ijSci.2390

24. Cosic I, Cosic D, Loncarevic I: Analysis of Ivermectin as Potential Inhibitor of SARS-CoV-2 Using Resonant Recognition Model. International Journal of Sciences, 2021; 10(1), 1-6, doi: 10.18483/ijSci.2433.

25. Cosic I, Drummond AE, Underwood JR, Hearn MTW: In vitro inhibition of the actions of basic FGF by novel 16 amino acid peptides. Molecular and Cellular Biochemistry, $1994 ; 130,1-9$

26. Krsmanovic V, Biquard JM, Sikorska-Walker M, Cosic I, Desgranges C, Trabaud MA, Whitfield JF, Durkin JP Achour A, Hearn MT: Investigation Into the Cross-reactivity of Rabbit Antibodies Raised against Nonhomologous Pairs of Synthetic Peptides Derived from HIV-1 gp120 proteins. J.Peptide Res, 1998; 52(5), 410-412.

27. Hearn MTW, Biquard JM, Cosic I, Krsmanovic V: Peptides Immunologically related to proteins expressed by a viral agent, having a sequence of amino acids ordered by means of protein informational method. US Patent 6, 294, 1742001.

28. Achour A, Biquard JM, Krsmanovic V, M'Bika JP, Ficheux D, Sikorska M, Cozzone AJ: Induction of Human Immunodeficiency Virus (HIV-1) Envelope Specific CellMediated Immunity by a Non-Homologus Synthetic Peptide. PLoS ONE, 2007; 11, 1-12, doi: 10.1371/journal.pone.0001214

29. Almansour N, Pirogova E, Coloe P, Cosic I Istivan T: Investigation of cytotoxicity of negative control peptides versus bioactive peptides on skin cancer and normal cells: a comparative study. Future Medicinal Chemistry, 2012; 4(12), 1553-1565.

30. Istivan T, Pirogova E, Gan E, Almansour N, Coloe P, Cosic I: Biological effects of a De Novo designed myxoma virus peptide analogue: Evaluation of cytotoxicity on tumor cells. Public Library of Science (PLoS) ONE, 2011; 6(9), 1-10. 